Research Article

\title{
Performance of Resource Management Techniques for Weather-Impacted Satellite Networks
}

\author{
Olugbenga Emmanuel Imole (iD) and Tom Mmbasu Walingo \\ School of Electrical Electronic and Computer Engineering, University of KwaZulu-Natal, Durban, South Africa \\ Correspondence should be addressed to Olugbenga Emmanuel Imole; oeimole@gmail.com
}

Received 26 July 2019; Accepted 25 October 2019; Published 1 December 2019

Academic Editor: Cong Pu

Copyright ( $\odot 2019$ Olugbenga Emmanuel Imole and Tom Mmbasu Walingo. This is an open access article distributed under the Creative Commons Attribution License, which permits unrestricted use, distribution, and reproduction in any medium, provided the original work is properly cited.

\begin{abstract}
Signals transmitted via satellite networks at high frequency in the $\mathrm{Ka}, Q$, and $V$ bands are susceptible to degradation due to rain attenuation. Adaptive transmission techniques are usually employed to mitigate the effect of rain and improve users' quality of service (QoS) but the effectiveness of these techniques hinges on the accuracy with which rain attenuation on the link is known. Commonly, most techniques rely on predicted attenuation along the link for selection of optimal transmission parameters. This paper proposes an efficient approach to predict the rain attenuation experienced by sources of multimedia connections in rainimpacted satellite networks. The proposed technique is based on three Markov models for widespread, shower, and thunderstorm rain events and predicts the attenuation experienced at different periods within the duration of a user's connection. It relies on an adaptive modulation and coding (AMC) scheme to dynamically mitigate rain attenuation and a call admission control (CAC) policy to guarantee the satisfaction of users' QoS requirements.
\end{abstract}

\section{Introduction}

The ability of satellites to offer ubiquitous coverage and large bandwidth is a unique advantage that enables them support large number of multimedia sources connected via fixed and/ or mobile architecture [1]. But, as we experience an increasing number of connected users, satellite networks now require larger bandwidths to support the huge traffic generated by users, majority of whom are accessing multimedia content/ services. This quest for increased capacity has resulted in migration to higher frequencies such as $\mathrm{Ka}, \mathrm{Q}$, and $\mathrm{V}$ bands, where larger bandwidths are available. While this migration addresses the capacity challenge, it also births the challenge to mitigate the effect of rain attenuation. Rain attenuation is a time-varying phenomenon that accompanies rain events. The attenuation produced during rain events usually depends on the intensity of the rain and may impact destructively on the quality of transmitted signals, especially in systems that operate at frequencies of $10 \mathrm{GHz}$ and beyond. When it occurs, rain attenuation provokes a violation of the quality of service (QoS) requirements of accepted/ongoing user connections. In addition, its occurrence may result in an inefficient utilization of allocated radio resources. In order to address these challenges, the implementation of highly efficient radio resource management (RRM) techniques such as link adaptation and call admission control (CAC) are inevitable [1]. However, the efficiency of these techniques in guaranteeing optimal performance of the network in the utilization of allocated resources as well as satisfaction of the QoS requirements of accepted connections is directly related to the accuracy with which the dynamics of rain attenuation are perceived. Prediction-based models of rain events have been developed and are employed to predict the rain attenuation on satellite links [2-4]. Based on these models, various techniques are developed to efficiently mitigate the rain attenuation experienced by users. But the efficiency of these techniques depends on the precision with which rain attenuation statistics are captured over time. For instance, the prediction methods employed in [5-8] estimate the rain attenuation that will be experienced during a user's connection without considering that the attenuation experienced during rain events is dynamic in time. Hence, when implemented, the proposed approach may result 
in inefficient utilization of allocated bandwidth as accepted connections may receive insufficient or excessive bandwidth allocation. For this reason, the development of dynamic models that predict the rain attenuation with time is deemed necessary. Rain attenuation models that are based on Markovian random processes have been developed in the literature [9-11]. Markov chain models for the satellite channel are developed from experimental data which have been acquired over a period of time. But the reliability of these models lies in the depth of the analysis of the data from which they have been developed, i.e., the definition of states and the number of states considered. Therefore, this paper develops a rain attenuation prediction model that can predict the rain attenuation experienced by users' connections at different intervals within the duration of their connection. We also develop a link adaptation procedure based on adaptive modulation and coding (AMC) to dynamically mitigate the attenuation experienced by users at each interval within the duration of their connection. The performance of the proposed method is then investigated by developing a CAC policy which ensures that users QoS requirements are satisfied for the entire duration of their connectivity.

The remainder of this paper is organized as follows: Section 2 is a brief review of some related works. In Section 3, the system model is presented. Section 4 describes the proposed CAC policy while results of investigation of the performance of the CAC policy are presented and discussed in Section 5. Finally, Section 6 presents our conclusions.

\section{Related Works}

In the recent literature, the feasibility of realizing an integrated and optimally performing satellite-terrestrial network is investigated. For instance, in $[12,13]$, the integration of fixed satellite service (FSS) and terrestrial cellular networks operating in the $m m$ Wave spectrum is studied. The authors show through coordinated BS scheduling and exploitation of certain characteristics of the $m m$ Wave spectrum that high spectral efficiency (SE) can be achieved within the regulatory bounds of the FSS. The authors in $[14,15]$ also investigate the trade-off between spectral efficiency and energy efficiency (EE) in hybrid satellite-terrestrial networks. It was found that the implementation of a controlled adjustment of key network performance indicators such as transmit power and overhead cost will facilitate higher energy and spectral efficiency in the integrated network.

Other authors in $[16,17]$ investigate solutions for improving physical layer performance of the satellite segment of the integrated network. The proposals demonstrate that high network capacity, throughput, and transmission efficiency can be achieved. These research efforts have all indicated that despite the existence of architectural and operational differences between satellite and terrestrial wireless networks, both networks can be successfully integrated into a single architecture. However, in order to optimize the performance of the integrated network, a deliberate commitment to mitigate the inherent challenges confronting individual networks is mandatory. This work, therefore, focuses on improving the overall performance of the satellite segment of the network.

Research efforts have also been directed at enhancing user experience on the integrated network. While some proposals are aimed at ensuring that accepted users are served with seamless and reliable connectivity, others focus on guaranteeing the satisfaction of users' individual quality of service (QoS) requirements throughout the period of their connection. For instance, the authors in $[18,19]$ address the issue of resource allocation for multicast multimedia content delivery to large number of users in an integrated satelliteterrestrial network by developing a resource allocation strategy that considers the time-varying condition of the satellite channel in the allocation of network resources. While aiming to guarantee users' QoS requirements and maximize resource utilization, users are organized into subgroups based on their current channel conditions; resources are then allocated to users in each subgroup depending on the modulation and coding scheme (MCS) that is appropriate and selected for transmission in their current channel states. However, determining the configuration of subgroups for each multicast session is a complex procedure and the effectiveness of this strategy remains to be tested in a duplex satellite communication scenario, which is very common to most contemporary applications. CAC policies are also proposed for the handling of multimedia connections in satellite networks. Traditional CAC policies proposed in [20-22] are inefficient in providing QoS guarantees to multimedia connections in contemporary satellite networks due mainly to their lack of consideration of the impact of the time-varying behaviour of satellite channel on users' experience. Other proposals for CAC in satellite networks [5-7], where rain fading is mitigated by the adaptation of forward error correction (FEC) codes only, to the rain attenuation dynamics are not optimal in performance. A basic yet dynamic approach to admission control and link state prediction and adaptation to mitigate rain attenuation in satellite networks is presented in [23]. Although the proposed approach yields promising results, a more robust modeling of the various rain conditions should be considered and used to test the performance of the proposed approach. Therefore, the implementation of highly dynamic and efficient techniques is still desirable. Furthermore, considering that rain attenuation phenomenon is dynamic in nature, its prediction and mitigation should be dynamic in order to realize efficient network performance. As a result of this, the operations of resource management policies such as CAC should be based on information on the selection of channel adaptation processes such as adaptive modulation and coding (AMC). This approach would aid a CAC's decision process with accurate information on channel conditions and transmission modes so that it can make good decision on the acceptance/rejection of connection requests. Based on this understanding, this work therefore proposes a CAC policy for satellite networks. Its major contributions are as follows:

(i) We present three Markov models for widespread, shower, and thunderstorm rains. In addition, we 
develop a model for predicting the rain attenuation experienced by users on the satellite network. This model regards the dynamic nature of rain attenuation and therefore predicts the attenuation experienced along the satellite link at successive periods (intervals) within the duration of each user's connection. From the results, an optimal number of prediction periods was determined.

(ii) We design a CAC policy based on game theory to provide QoS guarantee to real-time connections. The CAC is made more efficient through knowledge of the channel state and the current adaptation parameters (transmission modes) employed by the user to mitigate the attenuation caused by rain.

\section{System Model}

3.1. System Architecture. The architecture of the satellite network considered is of the form depicted in Figure 1. The network comprises a geostationary orbit satellite with bidirectional links-forward and return links. The satellite's forward link is broadcast-based and uses technologies similar to the second-generation Digital Video Broadcasting (DVB-S2) [24] standard to reach large number of users. The return link is employed by users to transmit information towards the satellite and its specifications conform to the second generation Digital Video Broadcasting Return Channel via Satellite (DVB-RCS2) standard defined in [25]. The networks also includes a large number of user terminals commonly referred to as return channel satellite terminals (RCSTs) and a gateway, which connects the satellite network to external networks such as the Internet or corporation networks. In this work, our interest is in the return link of the satellite segment, whose capacity is usually very small in comparison with that of the broadcast-based forward link. The return link's bandwidth is segmented using multiple frequency-time division multiple access (MF-TDMA) technique, such that network resources are available in the form of bandwidth-time units otherwise known as timeslots. Therefore, system capacity is allocated to each RCST in contiguous blocks of timeslots, the number of which is determined by the bandwidth requirements of the requested service(s).

3.2. Traffic Classes. The traffic generated by network connections may be broadly classified into real-time (RT) and nonreal-time (NRT) classes. Connections that belong to the real-time traffic class are characterized by stringent QoS requirements that must be satisfied in order to guarantee user satisfaction. Connections of voice and high-quality video services are classified as RT types because they require that their data rate, delay, and jitter specifications be respected throughout their connections. On the other hand, the NRT traffic, otherwise known as best effort (BE) traffic class, refers to nonsensitive traffic such as those of e-mail and file transfer, which, unlike RT traffic, are indifferent to delay and jitter. With reference to the DVB-RCS2 standard [25], connections may be classified based on the capacity request strategy required to provide capacity for connections. Five capacity request (CR) strategies are defined, namely,

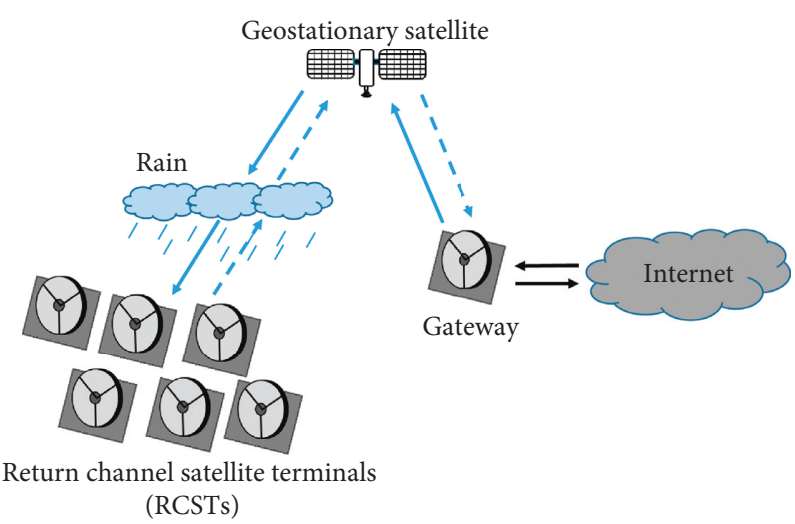

$\longrightarrow$ Forward link

-- Return link

FIgURE 1: Reference architecture.

continuous rate assignment (CRA), rate-based dynamic capacity (RBDC), volume-based dynamic capacity (VBDC), absolute volume-based dynamic capacity (AVBDC), and free capacity assignment (FCA). Only the first four strategies may be used for capacity requests depending on the service intended; the fifth is used to allocate unused capacity. All RT connections would require any one of CRA or RBDC to request capacity. While CRA would provide constant peak data rate (PDR) guarantee for connections of voice and highquality video, RBDC on the other hand would provide data rate guarantees for sources with variable bitrate (VBR) profiles. The other strategies are employed to provide capacity for NRT sources. Connections of both RT and NRT traffic classes are considered in this work.

3.3. Weather-Impacted Satellite Channel. As indicated in Figure 1, signals transmitted at frequencies beyond $10 \mathrm{GHz}$ are susceptible to degradation due to rain attenuation. Moreover, the magnitude of the attenuation experienced depends largely on the intensity of the rain. Based on its intensity $(R)$, a rain event may be classified into one of four types, namely, drizzle $(D)$, widespread $(\mathrm{W})$, shower $(\mathrm{S})$, and thunderstorm $(\mathrm{T})$ with each type producing unique levels of attenuation [26, 27]. The intensities of the different classes of rain are presented in Table 1.

Note that the derived models adopted in this work feature different rain intensities for a particular terrain as obtained in $[28,29]$. Markov models are popular for modeling rain events and are usually developed from measured rainfall data obtained over a specified period of time. The model is usually described by a steady-state and transition probability matrix, both of which are evaluated from the measured rainfall data [11,30-32]. In [30], a fourstate Markov model for rain events is developed. The model simply considers a rain event as transition events among the four types of rain. However, recent studies on measured rainfall data in Durban, South Africa, have shown that a rain event is usually a succession of rains of different intensities which occur as a result of succession of cloud arrivals $[28,29]$. Aligning with these studies and the classification shown in Table 1, any rain event may, therefore, be classified 
TABLE 1: Classification of rain events.

\begin{tabular}{lc}
\hline Rain type & Rain rate $(R)$ \\
\hline Drizzle $(D)$ & $R<5 \mathrm{~mm} / \mathrm{hr}$ \\
Widespread $(W)$ & $5 \mathrm{~mm} / \mathrm{hr} \leq R<10 \mathrm{~mm} / \mathrm{hr}$ \\
Shower $(S)$ & $10 \mathrm{~mm} / \mathrm{hr} \leq R<40 \mathrm{~mm} / \mathrm{hr}$ \\
Thunderstorm $(T)$ & $R \geq 40 \mathrm{~mm} / \mathrm{hr}$ \\
\hline
\end{tabular}

according to the peak rain intensity recorded during that event. Hence, three Markovian rain models are defined for this work. The first is the widespread rain model in which the values of rain intensities were recorded during the event range between those of drizzle and widespread rains. The second is the shower rain model which records a maximum rainfall intensity value which lies between 10 and $40 \mathrm{~mm} / \mathrm{hr}$ during the period of the event. It should be noted that this event may also record rain intensity values that lie within widespread and drizzle. The third model, referred to as the thunderstorm rain model, models a rain event that records a maximum rainfall intensity value that is greater than $40 \mathrm{~mm} / \mathrm{hr}$. This type of event would see a random succession of drizzle, widespread, shower, and thunderstorm rain. The transition diagrams of the widespread, shower, and thunderstorm rain models are shown in Figures 2(a)-2(c). Each model's steady-state and transition probabilities matrices are presented in Table 2 in which elements $P_{i j}$ of the transition probability matrices indicate the probability of transitioning from a current state $i$ to another state $j, i, j \in\{D, W, S, T\}$. The elements $W_{i}$ of each transition matrix denote the steadystate probability of the $i$ th state of each Markov model, where $i \in\{D, W, S, T\}$. These values of $P_{i j}$ and $W_{i}$ were derived from measured rain data in [28], but values obtained from rain data for other areas are still applicable. Note that the derived models feature different rain intensities for a particular terrain as obtained in $[11,28]$.

3.4. Prediction of Channel Conditions. In order to ensure efficient and reliable transmission of signals over the satellite channel, rain attenuation should be mitigated as the channel state evolves. However, determining how often the channel state should be estimated without introducing unnecessary latency on the link is a challenge. In [5-8], this challenge is confronted through the prediction of rain attenuation that may be experienced over the period of any connection. By predicting the attenuation, the resources required to support a connection and guarantee the satisfaction of its QoS requirements under such condition can be estimated and allocated to it. However, considering that rain attenuation is a dynamic phenomenon, the static allocation of resources may result in poor utilization of network resources if excessive or insufficient resources are allocated to the connection. We therefore propose a dynamic prediction of the rain attenuation experienced for different periods within the duration of a connection.

Let us assume that the duration $T_{s}$ of each connection required to be setup in the network is known a priori. The process for predicting the rain attenuation experienced by any connection begins by the partitioning of $T_{s}$ into $N$ periods, each spanning a duration $\Delta t$, where $T_{s}=N \Delta t$ as shown in Figure 3. The state of the channel is predicted for each period $n,\{n=1,2,3, \ldots, N\}$ in $T_{s}$ by the Markov chain model described previously. Since the attenuation is a time-varying phenomenon, its value is estimated every period within the connection's duration and the maximum attenuation for each period is a function of the rain rate at that time. The maximum rain rate for any period $n$ is denoted by $R_{\max , n \Delta t}$. Using the estimated rain rate and other parameters such as frequency, elevation angle, polarization, and effective path length, the rain attenuation $A_{R, n \Delta t}$ within the period $n$ may then be evaluated as follows:

$$
A_{R, n \Delta t}=k R_{\max , n \Delta t} \alpha L_{E},
$$

where $k$ and $\alpha$ are frequency-dependent coefficients and $L_{E}$ is the effective path length between the satellite and antenna of the receiver. Free space loss $\left(A_{F S}\right)$ is another major source of attenuation and its impact is also considered. Its value may be estimated using the following relation:

$$
A_{F S}=20 \log (4 \pi d / \lambda)
$$

where $d$ is the distance between the antenna and the satellite and $\lambda$ denotes the wavelength. Therefore, the total attenuation $A_{T, n \Delta t}$ within any period $n$ of a connection is obtained as the sum of the attenuation due to rain and free space loss, i.e.,

$$
A_{T, n \Delta t}=A_{R, n \Delta t}+A_{F S} \mathrm{~dB} \text {. }
$$

\subsection{Selection of Transmission Modes and Dynamic Resource} Calculation. In order to facilitate an efficient allocation and optimal utilization of network resources by accepted connections, a dynamic resource allocation strategy is adopted. This strategy allocates resources to support user connections based on the evolution of the channel states and the transmission parameters employed to mitigate the attenuation experienced in each state. Each connection's channel quality is indicated by its receiver's signal-to-noise ratio (SNR), a parameter that relates the strength of the received signal to the attenuation and noise present on the channel. The $\mathrm{SNR}_{n \Delta t}$ for each period $n,\{n=1,2,3, \ldots, N\}$ in $T_{s}$ is estimated by

$$
\mathrm{SNR}_{n \Delta t}=P_{T}+G_{T}-A_{T, n \Delta t}+G_{R}-T-K-S \mathrm{~dB},
$$

where $P_{T}$ denotes the transmit power, $G_{T}$ and $G_{R}$ are the antenna gains at the transmitter and receiver, respectively, $T$ denotes the effective noise temperature, $K$ symbolizes the Boltzmann constant, and $S$ is the symbol transmission rate. Furthermore, $M$ transmission modes are defined based on the number of channel states. Each mode $m,\{m=1,2,3, \ldots, M\}$ comprises the modulation format and coding rate (MODCOD) that optimizes the spectral efficiency in a specific channel state. However, the spectral efficiency, $\eta_{m}$, of any transmission mode, $m$, selected to mitigate the attenuation experienced in any channel state will determine the maximum achievable bitrate in that state. The maximum achievable bitrate $b_{m, n \Delta t}$ in the $n$th period of $T_{s}$ is, therefore, a variable that depends on the channel state and selected transmission mode. It may be estimated as follows: 

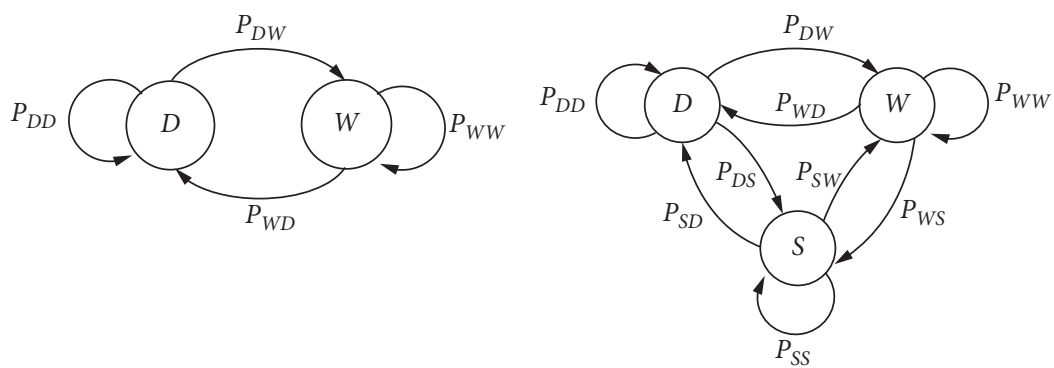

(a)

(b)

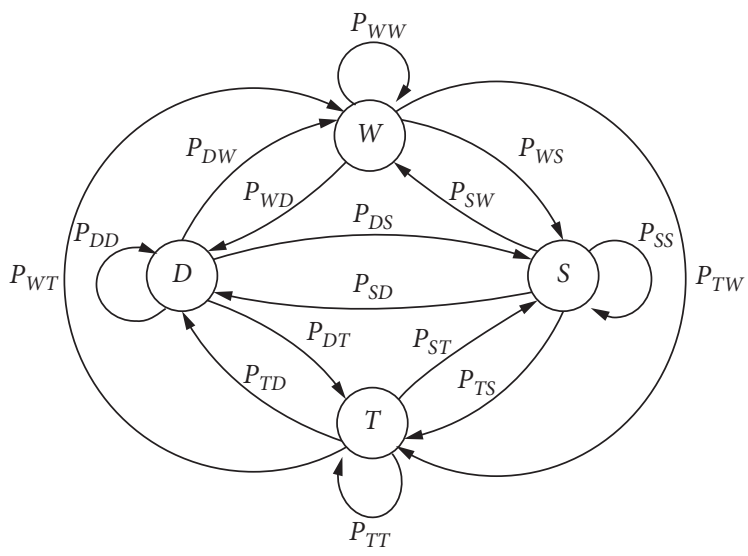

(c)

Figure 2: Markov models for different rain events. (a) Widespread rain event. (b) Shower rain event. (c) Thunderstorm rain event.

TABLE 2: Steady-state and transition probability matrices [28].

Rain event Transition probability matrix $(P)$ Steady-state probability matrix $(W)$



$$
b_{m, n \Delta t}=\left\{\begin{array}{l}
0: \text { if } \mathrm{SNR}_{n \Delta t}<\Gamma_{\min }, \\
\eta_{1} S: \text { if } \Gamma_{\min } \leq \mathrm{SNR}_{n \Delta t}<\Gamma_{1} \\
\eta_{1} S: \text { if } \Gamma_{1} \leq \mathrm{SNR}_{n \Delta t}<\Gamma_{2}, \\
\vdots \\
\eta_{1} S: \text { if } \Gamma_{M-1} \leq \mathrm{SNR}_{n \Delta t},
\end{array}\right.
$$

where $\Gamma_{\min }$ denotes the minimum SNR threshold and $\Gamma_{m}\{m=1,2, \ldots, M\}$ denotes the SNR thresholds for the $m$ th mode of the AMC scheme. The transmission modes adopted for the widespread, shower, and thunderstorm rain events are presented in Table 3. In each case, the rain attenuation experienced is indicated by different levels of rain intensity. 


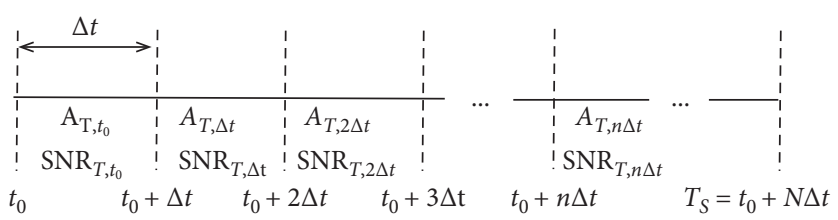

Figure 3: Rain attenuation prediction model.

TABle 3: AMC modes.

\begin{tabular}{|c|c|c|c|}
\hline Transmission mode & MODCOD & $R(\mathrm{~mm} / \mathrm{hr})$ & Spectral efficiency (bits/s/Hz) \\
\hline \multicolumn{4}{|l|}{ Widespread Event } \\
\hline 1 & QPSK 1/2 & $R<5$ & 1.0 \\
\hline 2 & 8PSK $1 / 2$ & $5 \leq R<10$ & 2.0 \\
\hline \multicolumn{4}{|l|}{ Shower Event } \\
\hline 1 & QPSK 1/2 & $R<5$ & 1.0 \\
\hline 2 & QPSK $3 / 4$ & $5 \leq R<10$ & 1.5 \\
\hline 3 & 8PSK $1 / 2$ & $10 \leq R<40$ & 2.0 \\
\hline \multicolumn{4}{|l|}{ Thunderstorm Event } \\
\hline 4 & QPSK 1/2 & $R>40$ & 1.0 \\
\hline 3 & QPSK 3/4 & $10 \leq R<40$ & 1.5 \\
\hline 2 & 8PSK $1 / 2$ & $5 \leq R<10$ & 2.0 \\
\hline 1 & 8PSK 6/7 & $R<5$ & 2.57 \\
\hline
\end{tabular}

Considering that the maximum bitrate that can be achieved by each connection differs for all channel states, the network capacity that should be allocated to each connection will also vary with the evolution of channel states. To determine the capacity required to accept any connection request, we estimate the $p$ th percentile of the channel states that have been predicted for all $N$ periods within its duration, $T_{S}$, and evaluate the capacity required to support the connection in the channel state obtained. The total attenuation $\left(A_{T, n \Delta t}^{p \%}\right)$ and $\mathrm{SNR}\left(\mathrm{SNR}_{T, n \Delta t}^{p \%}\right)$ for the obtained state may be estimated using equations (3) and (4), respectively, while the maximum bitrate achievable $b_{m, n \Delta t}^{p \%}$ in that state may also be obtained from equation (5). The capacity, $C_{R, m}$ (number of timeslots), that is required and should be allocated to accept the connection if its bitrate requirement is $B_{R} K$ bits/s and when a state $m$ is evaluated as the $p$ th percentile of all predicted states may be obtained from the following:

$$
C_{R, m}=\left\lceil\frac{B_{R}}{b_{m, n \Delta t}^{p \%}}\right\rceil .
$$

The $x$ operator indicates the largest integer greater or equal to $x$. For the sake of clarity, the range of percentile $p$ is defined as $1<p \leq 100$.

\section{Proposed Call Admission Control}

4.1. Call Admission Control. Let the capacity of the satellite network be denoted by $C_{T}$ and the network supports traffic generated by multiple connections of RT and NRT sources. If $X$ RT and $Y$ NRT connections are currently established in the network, we denote the total capacity allocated to all $X$ ongoing RT connections by $C_{\mathrm{RT}}$ and the total capacity allocated to all $Y$ ongoing NRT connections by $C_{\mathrm{NRT}}$. Then, we can express $C_{\mathrm{RT}}$ and $C_{\mathrm{NRT}}$ as follows:

$$
\begin{aligned}
C_{\mathrm{RT}} & =\sum_{x=1}^{X} \sum_{m=1}^{M} C_{\mathrm{RT}}^{x, m} \rho_{m}, \\
C_{\mathrm{NRT}} & =\sum_{y=1}^{Y} \sum_{m=1}^{M} C_{\mathrm{NRT}}^{y, m} \rho_{m},
\end{aligned}
$$

where $C_{\mathrm{RT}}^{x, m}$ is the capacity that is currently allocated to the $x$ th ongoing RT connection employing AMC mode $m$ and $C_{\mathrm{NRT}}^{y, m}$ is the capacity currently allocated to the $y$ th ongoing NRT connection employing AMC mode $m . \rho_{m}$ is the steadystate probability of the $m$ th state of the channel. Due to the stringent QoS requirements of RT connections, the proposed CAC policy prioritizes the acceptance of RT connections over their NRT counterparts. However, a maximum capacity of $\phi C_{T}$ may only be utilized by all accepted RT connections depending on the priority assigned to them. The parameter $\phi,\{0 \leq \phi \leq 1\}$ indicates the priority of RT connections and may also be employed to tune the performance of the proposed CAC policy.

The proposed CAC policy is presented in 1 below. It is initiated at the arrival of a new RT or NRT connection request.

As indicated in the algorithm, a new connection request from a RT or NRT source will be accepted if

$$
C_{T}-C_{\mathrm{RT}}-C_{\mathrm{NRT}} \geq C_{R},
$$

where $C_{R}$ is the capacity requested by the new connection. This condition is the basic requirement that must be met before the acceptance of new connection requests. If the new connection request is that of a NRT traffic source and the condition in equation (8) is not satisfied, the connection request is rejected. However, if the connection request had originated from a RT traffic source, then the following two actions are taken. Firstly, the RT connection is accepted if the 


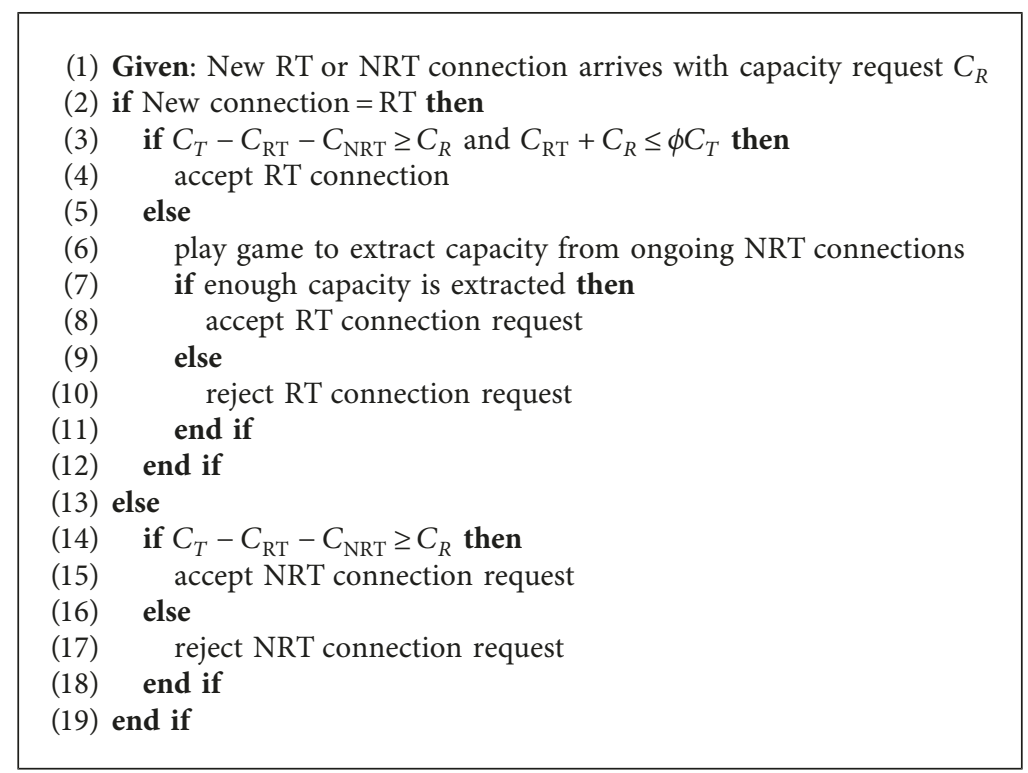

Algorithm 1: CAC algorithm.

bandwidth threshold for RT connections has not been exceeded, i.e.,

$$
C_{\mathrm{RT}}+C_{R} \leq \phi C_{T}
$$

The second action is initiated only if the condition in equation (9) is not satisfied. This action will investigate the possibility of extracting capacity from ongoing NRT connections by degrading the capacity of one or more of those connections. Therefore, the connection request will be accepted only if the available and extracted capacity can support its requested capacity. Otherwise, it is rejected. In the bandwidth extraction process, it is ensured that the minimum capacity requirement of all NRT connections is respected and guaranteed. This bandwidth extraction process is described in the following subsection.

4.2. Bandwidth Extraction Procedure. The concept of games usually derives from phenomenon where individuals (players) are in competition for resources (network capacity) and employ different strategies to maximize their payoffs (utilities). There is a competition for resources between RT and NRT connections as both classes of traffic seek to be allocated maximum capacity. This is the motivation for the adoption of the concept of game theory to model this competition and the bandwidth extraction procedure. The bandwidth extraction procedure follows the concept of a two-person noncooperative game and it is initiated when the condition in equation (9) is not satisfied. Its objective is to extract capacity from existing NRT connections whose current allocated capacity is greater than their minimum capacity requirement, $C_{\min }$. The game is defined by a tuple $G=[N, S, U]$, where $N$ denotes the players involved in the game, $S$ denotes a set of all possible actions or strategies that may be taken by the players and $U$ denotes the utility or satisfaction enjoyed by the players. In this context, a player refers to any of the connections of ongoing NRT connections as well as the new NRT connection requesting to be accepted into the network. Each of these connections will enjoy a utility which is a function that is directly linked to the strategy its adopts. In the subsections that follow, the utility functions of the ongoing RT and NRT connections as well as the possible strategies they might adopt are elucidated.

4.2.1. Utility Function. A connection's utility is a function that indicates the degree of satisfaction it enjoys and is related to the capacity allocated to it. As in [33], the utility is expressed as a universal sigmoid function, with unique parameters for RT and NRT traffic classes. When estimated, the utility of any RT or NRT connection is a value that lies in the domain $[0,1]$. Given that the capacity requirement of any RT or NRT connection who is currently employing the $m$ th transmission mode is denoted by $C_{\mathrm{RT}}^{m}$ and $C_{\mathrm{NRT}}^{m}$, respectively, then the utility enjoyed by any of their connections may be evaluated in relation to the amount of resources, $C$, that is allocated to it. This is expressed as follows:

$$
U(C, m)= \begin{cases}\frac{1}{e^{-G_{\mathrm{RT}}\left(C-C_{\mathrm{RT}}^{m}\right)} ;} & \mathrm{RT}, \\ \frac{1}{1+B e^{-G_{\mathrm{NRT}} C_{\mathrm{NRT}}^{m}}+D ;} & \mathrm{NRT},\end{cases}
$$

where the parameters $G_{\mathrm{RT}}$ and $G_{\mathrm{NRT}}$ indicate the steepness of the utility curve for RT and NRT connections around $C$ while $B$ and $D$ are constants taken as 1.5 and -0.4 respectively [33]. Since RT connections require the provisioning of their peak data rate capacity for QoS satisfaction, i.e., $C=C_{\mathrm{RT}}^{m}=\mathrm{PDR}$, the utility of all accepted $\mathrm{RT}$ connections is always equal to unity (equation (10)).

4.2.2. Payoffs. The action taken by players during the capacity extraction game results in different utilities for all 
players. Therefore, the utility enjoyed by players when an action is taken is referred to as the payoff and is depicted by a matrix, $U$. Each element $\left\{U_{n q}, U_{n}\right\}$ in $U$ denotes the payoff $U_{n q}$ of ongoing NRT connections when $b_{q}$ resources are extracted from them and the resulting payoff $U_{n}$ for a new connection when it either accepts or rejects the extracted resources. The acceptance is based on whether the available resources and extracted resources are sufficient to meet the capacity requirements of the new connection. The payoff matrix $U$ is expressed as follows:

$$
U=\left[\begin{array}{cc}
U_{b_{1}}, U_{A} & U_{b_{1}}, U_{R} \\
U_{b_{2}}, U_{A} & U_{b_{2}}, U_{R} \\
U_{b_{3}}, U_{A} & U_{b_{3}}, U_{R} \\
\vdots & \\
U_{b_{Q}}, U_{A} & U_{b_{Q}}, U_{R}
\end{array}\right],
$$

where $\left(U_{n q}\right)$ the payoff for ongoing NRT connections when $b_{q}$ resources have been extracted from them can be estimated by the following equation:

$$
U\left(C_{\mathrm{NRT}}^{m}-b_{q}\right)=\frac{1}{1+B e^{-G_{\mathrm{NRT}}\left(C_{\mathrm{NRT}}^{m}-b_{q}\right)}},
$$

and the payoff $U_{n}$ of the new connection when it accepts or rejects the extracted capacity allocated to it is given by

$$
U_{B}= \begin{cases}U_{A}=1 ; & \text { if accepted, } \\ U_{R}=0 ; & \text { otherwise. }\end{cases}
$$

4.2.3. Game Strategies. The game played aims to find a number of NRT connections (if any) whose capacity may be degraded in order to release additional capacity to support a new RT connection request. However, the capacity degradation process is regulated by the utility of players. Each player is capable of adopting a number of strategies, one at a time, depending on the capacity available. The strategy $S_{n}$ that may be adopted by a new connection is defined by a set of actions as follows:

$$
S_{n}=\left\{B_{A}, B_{R}\right\}
$$

where $B_{A}$ and $B_{R}$, respectively, denote acceptance and rejection of the available capacity after additional capacity has been extracted from ongoing NRT connections, respectively. On the other hand, the strategies adopted by the ongoing NRT connections is defined by a set $S_{n q}$, based on the quantity $q$ of resources extracted by degrading a number of ongoing NRT connections without violating their minimum capacity requirements. The strategy set $S_{n q}$ of ongoing NRT connections is defined as

$$
S_{n q}=\left\{b_{0}, b_{1}, \ldots, b_{Q-1}, b_{Q}\right\},
$$

where $b_{q}\{q=1,2, \ldots, Q\}$ denotes the action in which $q$ resources have been extracted from ongoing NRT connections and $Q$ denotes the maximum resources that may be extracted from NRT connections.
4.3. Bandwidth Degradation. As indicated previously, the bandwidth degradation activity is initiated in order to extract bandwidth from ongoing NRT connections whose allocated capacity is greater than their minimum capacity requirement. In order to formulate the bandwidth degradation procedure, we assume that there are $K$ ongoing NRT connections in the network. Let the maximum and minimum capacity requirements of the $k$ th NRT connection $\{k=1,2,3, \ldots, K\}$ be denoted by $C_{\mathrm{NRT}}^{\max , k}$ and $C_{\mathrm{NRT}}^{\min , k}$, respectively. In the bandwidth degradation procedure, a maximum bandwidth capacity of $C_{\text {degr }}$ may be extracted from an NRT connection during each degradation episode provided that its minimum bandwidth capacity requirement will not be violated. We define a row matrix, $D$ of $K$ elements such that each element $C_{\mathrm{NRT}}^{k}$ of $D$ denotes the current capacity allocated to the $k$ th ongoing NRT connection as follows:

$$
D=\left\{C_{\mathrm{NRT}}^{1} \quad C_{\mathrm{NRT}}^{2} C_{\mathrm{NRT}}^{3} \cdots C_{\mathrm{NRT}}^{k}\right\} .
$$

The value of $C_{\text {degr }}$ indicates that each NRT connection's bandwidth capacity may only be degraded in $\tau$ degradation episodes throughout its lifetime if its minimum bandwidth capacity requirement will not be violated. $\tau$ may be estimated by

$$
\tau=\frac{C_{\mathrm{NRT}}^{\max }-C_{\mathrm{NRT}}^{\min }}{C_{\mathrm{degr}}} .
$$

After each bandwidth degradation episode, the $k$ th NRT connection's bandwidth capacity allocation may be estimated by

$$
C_{\mathrm{NRT}}^{k}=C_{\mathrm{NRT}}^{\max }-d C_{\mathrm{degr}},
$$

where $d=0,1,2, \ldots, \tau$ is the number of degradation episodes in which the connection's bandwidth capacity has been extracted. The bandwidth degradation algorithm is presented in 2 .

\section{Simulation Results and Discussion}

We investigate the performance of the proposed policy using an event-driven simulator. The parameters set for the simulation are highlighted in Table 4. The performance measures of the investigation are the call blocking probability (CBP), throughput, and utilization factor. The CBP for each traffic class is obtained as the ratio of the total number of connections requests of a particular traffic class that was accepted to the total number of connection requests of that traffic class that was generated. We also define the throughput as the total bitrate achieved by ongoing connections on the network. The utilization factor is used to investigate the efficiency with the available network resources are utilized by accepted connections. It is determined by the ratio of total number of occupied timeslots to the total number of timeslots in the network.

5.1. Call Blocking Probability (CBP). The CBP of connections of RT and NRT traffic sources during widespread, shower, 


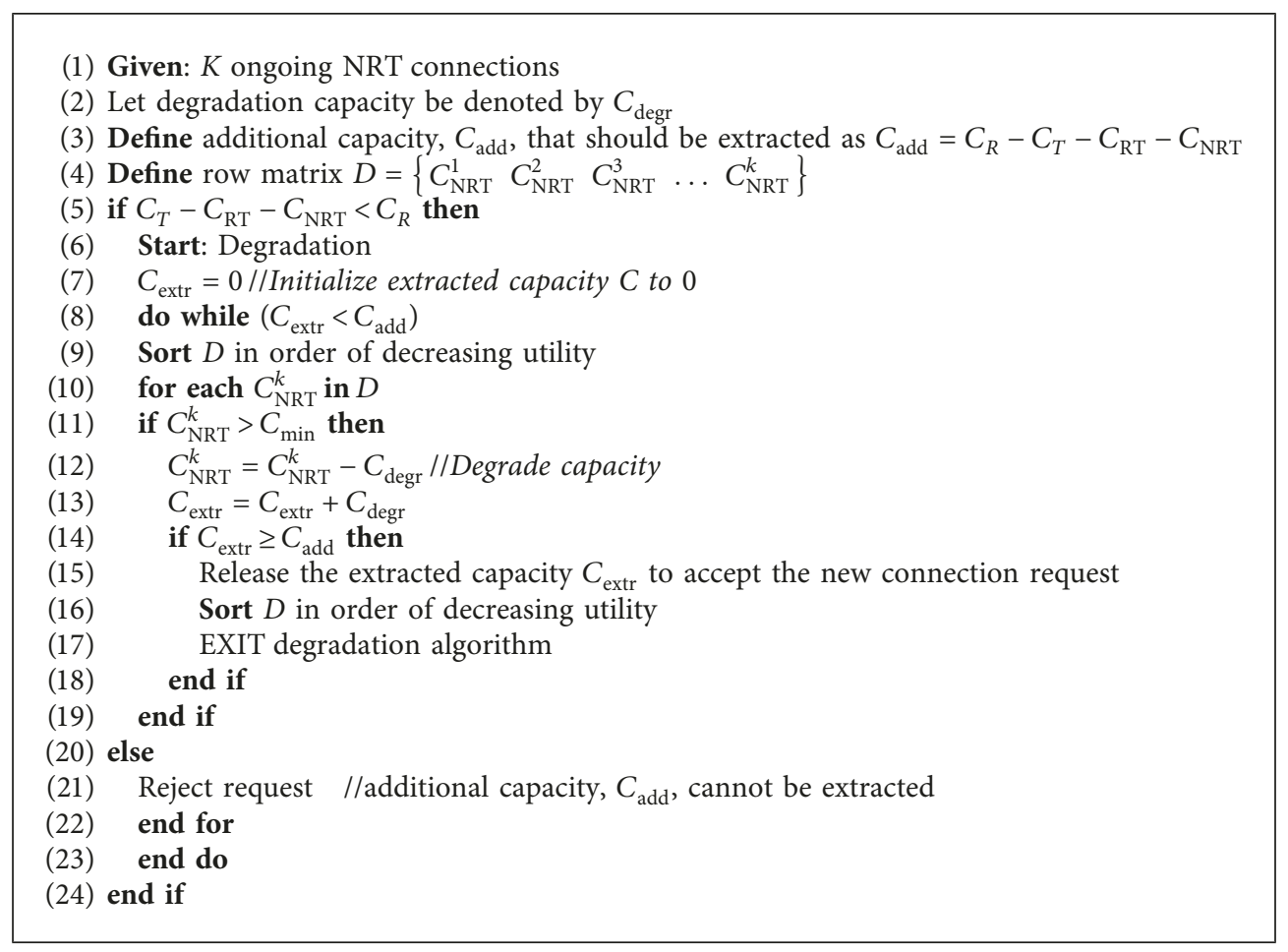

Algorithm 2: Bandwidth degradation algorithm.

and thunderstorm events are presented in Figures 4-6, respectively. They are presented as a function of the system load. In each of these figures, the CBPs are obtained for three different scenarios. The first is when the attenuation experienced by the user is predicted once ( 1 period) for the entire duration of the connection. In the second scenario, the attenuation is predicted for two intervals within the duration of the user's connection (2 periods), while in the last scenario, the attenuation is predicted for four intervals within the duration of each user's connection (4 periods). It can be seen from the results that during each rain event and for each of the prediction periods considered, RT connections enjoy lower CBPs that their NRT counterparts. It is also interesting to note from these results that the CBP experienced by connections of the two traffic classes decreases as the number of prediction intervals increases. This trend can be attributed to the increased frequency of the adaptation of transmission parameters while the number of periods is increased. Consequently, each traffic class experiences lower CBPs as the number of prediction intervals is increased and network resources are more efficiently allocated and utilized. In Figure 7, the CBPs of RT connections during widespread, shower, and thunderstorm events are compared at a system load value of 10 . This result shows that the best CBP values recorded under each rain event were at the 4-period prediction scenario. Thus, the CBP decreases as the number of prediction periods is increased.

5.2. Throughput. The network throughput during widespread, shower, and thunderstorm rain events and for different prediction periods are presented in Figures 8-10, respectively. From the results obtained for each rain event, the throughput can be seen to increase with the system load.
TABLE 4: Simulation parameters.

\begin{tabular}{lc}
\hline Parameters & Values \\
\hline System capacity & $32 \mathrm{Mbits} / \mathrm{s}$ \\
Air interface & MF-TDMA \\
Number of timeslots & 1000 \\
System load & $10-50$ \\
Call duration (exponential) & $600 \mathrm{~s}$ \\
Bitrate of RT sources & $384 \mathrm{Kbits} / \mathrm{s}$ \\
Bitrate of NRT sources $\left(C_{\min }\right)$ & $384 \mathrm{Kbits} / \mathrm{s}$ \\
Minimum bitrate of NRT sources & $192 \mathrm{Kbits} / \mathrm{s}$ \\
Degradation capacity $\left(C_{\text {degr }}\right)$ & $64 \mathrm{Kbits} / \mathrm{s}$ \\
Number of intervals $(\Delta t) /$ connection & $1,2,4,10$ \\
\hline
\end{tabular}

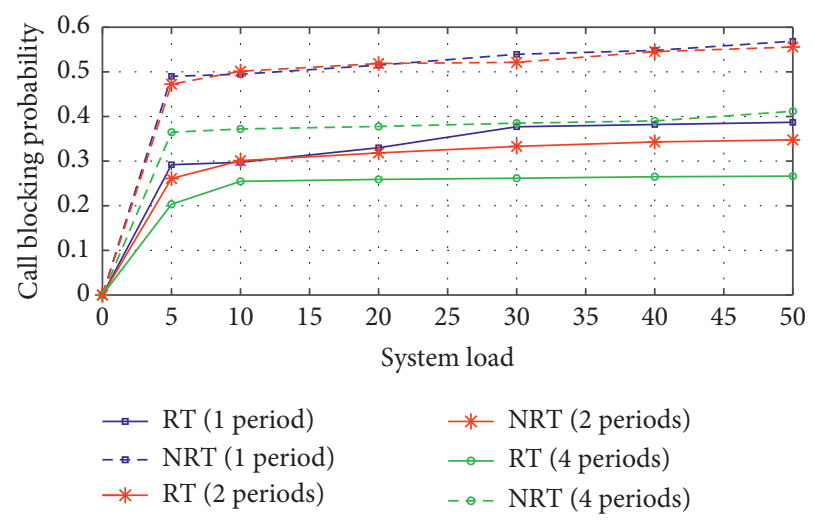

FIGURE 4: Call blocking probability (widespread rain).

In addition, the throughput achieved during all three rain events is similar at low loads, but as the load increases, slight differences in the throughput values are observed. However, 


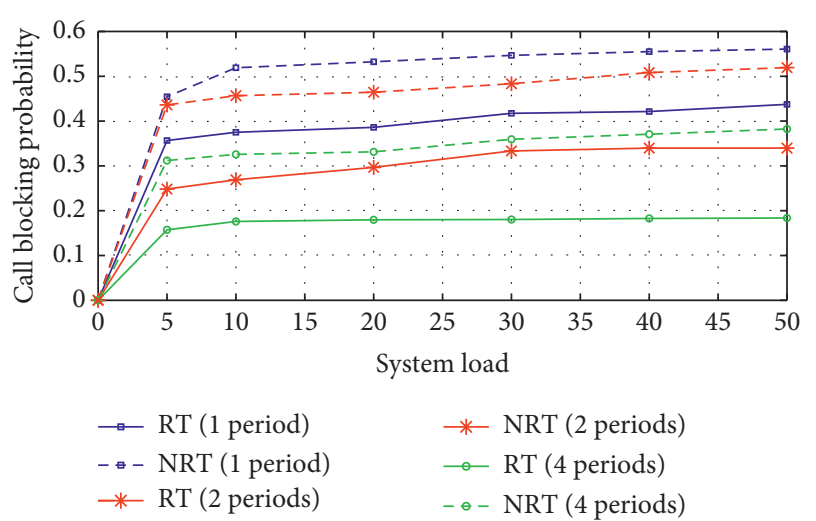

Figure 5: Call blocking probability (shower rain).



Figure 6: Call blocking probability (thunderstorm rain).

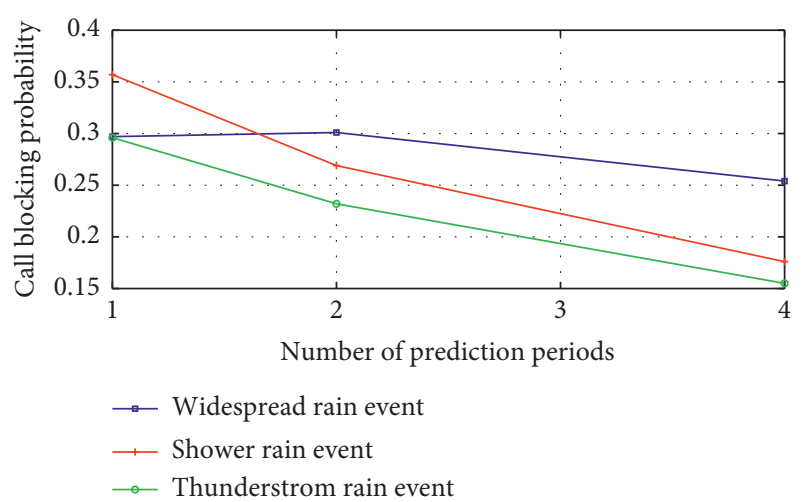

FIGURE 7: Comparison of call blocking probability.

the highest throughput recorded for all three events can be seen to occur during the 4-period prediction scenarios. This further underscores the fact that dynamic link adaptation at periodic intervals within the period of connection yields lower CBP and consequently higher throughput. In Figure 11, the network throughput at the system load value of 10 is compared for different rain events and number of prediction periods. It can also be observed from the plots that the best throughput recorded during the three rain events occurs during the 4-period prediction scenarios.

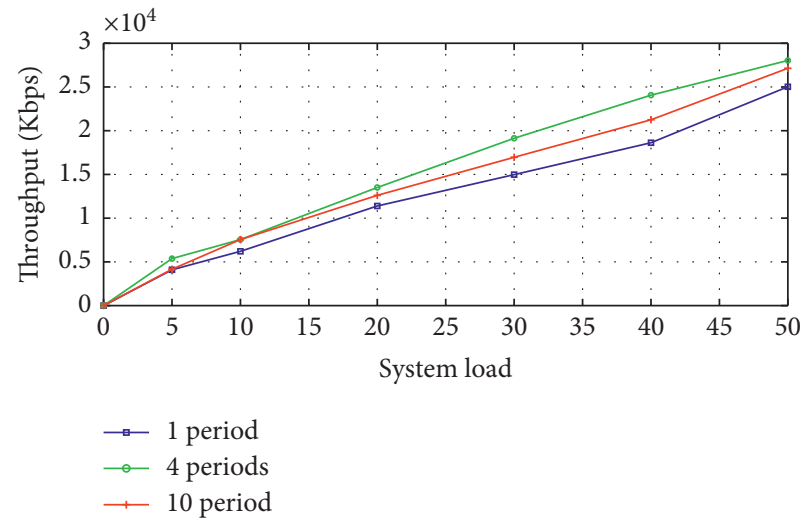

Figure 8: Throughput (widespread rain event).

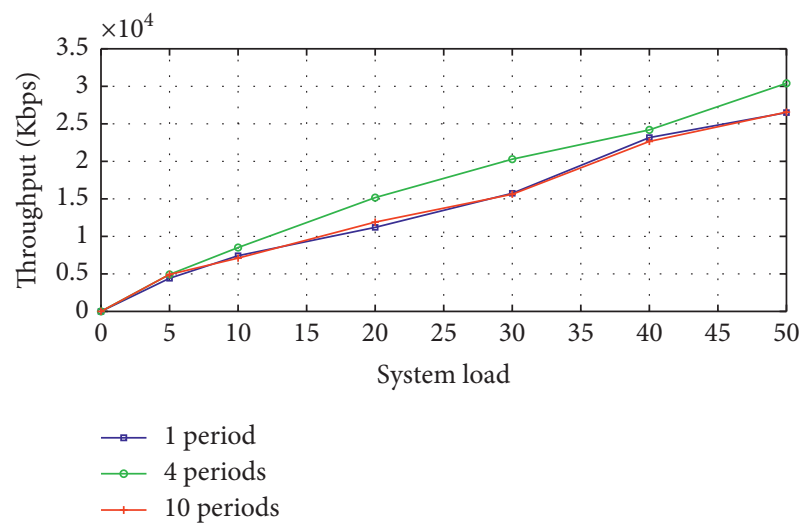

FIGURE 9: Throughput (shower rain event).

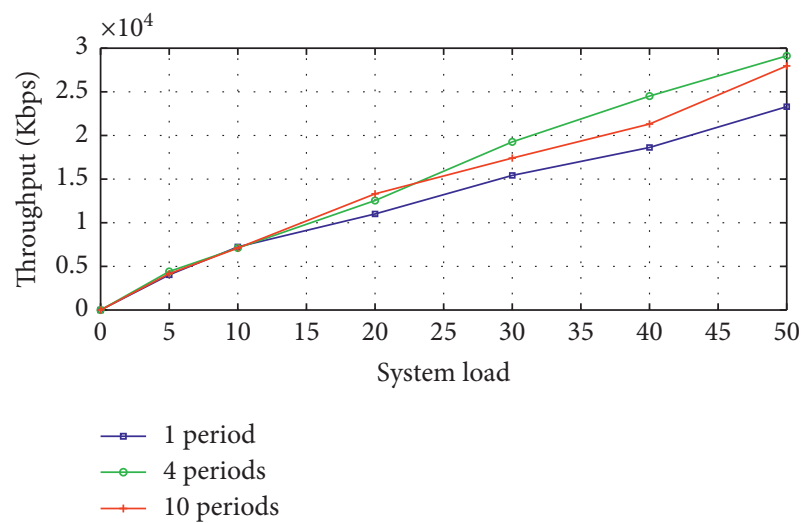

Figure 10: Throughput (thunderstorm rain event).

Thus, with few or too many prediction periods, a high throughput is not guaranteed. In this case, implementing a 4-period prediction of connections channel conditions seems to be optimal.

5.3. Utilization Factor. The capacity utilization achieved by the proposed policy during widespread, shower, and thunderstorm rain events is compared under different prediction intervals. In Figures 12-14, the utilization factors 


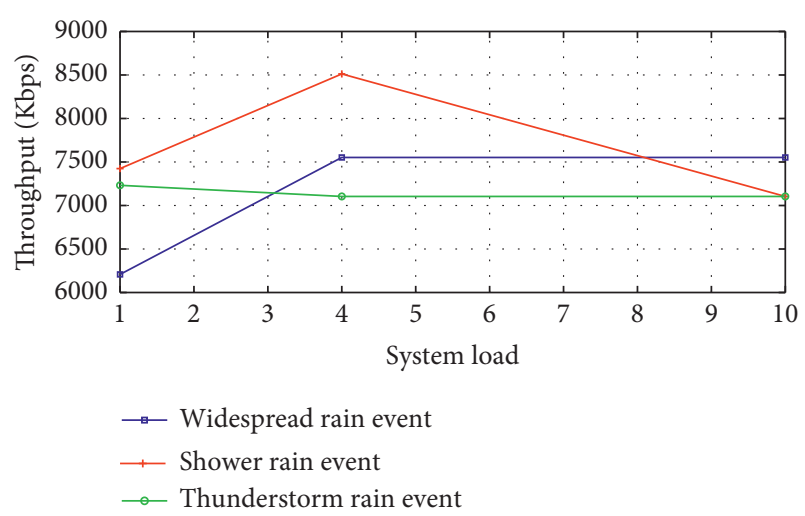

FIgURE 11: Comparison of throughput.

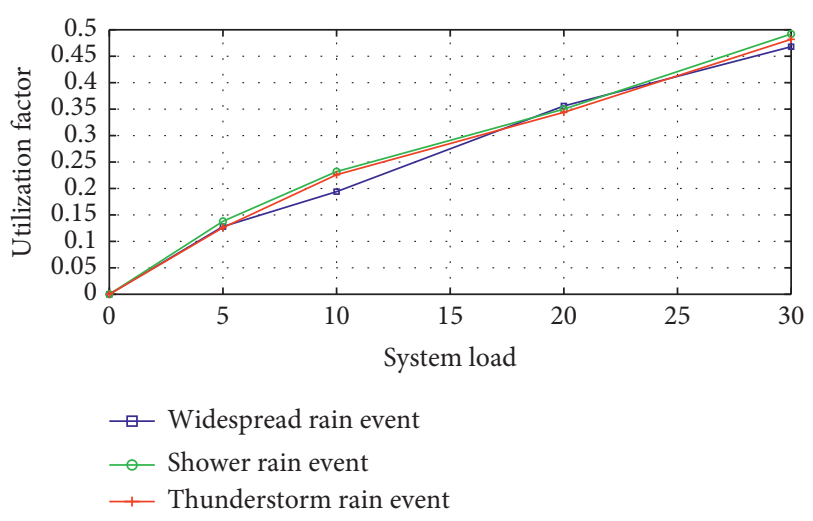

FIGURE 12: Utilization (one-period prediction).

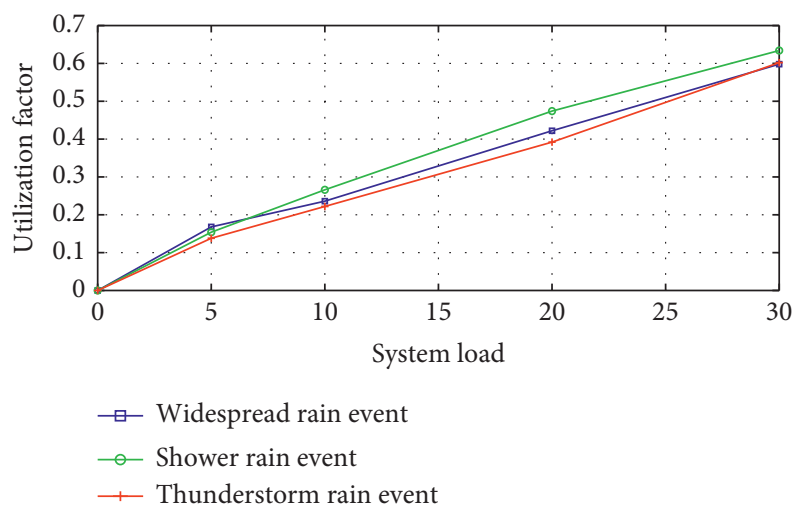

FIgURE 13: Utilization (four-period prediction).

under 1-, 4-, and 10-period predictions are shown. These also show that during all rain events, the best capacity utilization is achieved when the 4-period prediction scheme is implemented. This agrees with conclusions drawn from the CBP and throughput results previously presented. The prediction of channel states at four periods within the duration of each user's connection is optimal and achieves best network performance. A comparison of the different utilization factors obtained at system load of 10 under the three channel states also confirms this (Figure 15).

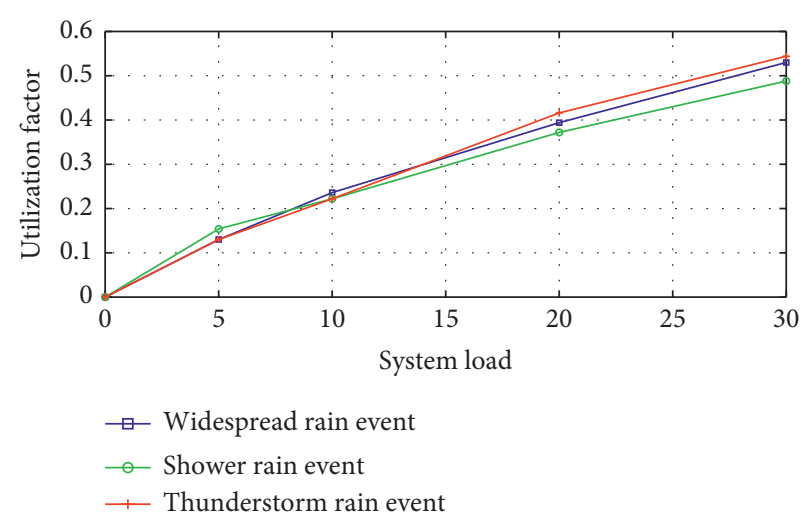

FIGURE 14: Utilization (ten-period prediction).

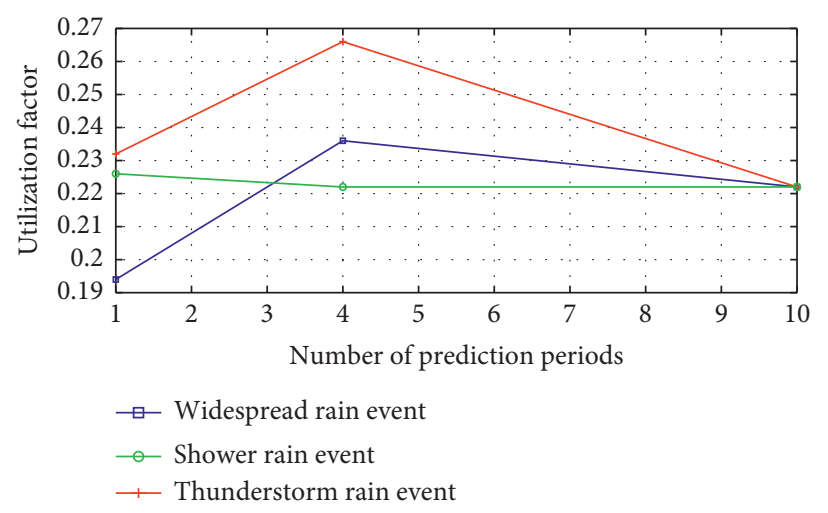

FIgURE 15: Comparison of utilization factor.

\section{Conclusions}

This work proposes an efficient admission control policy for providing QoS guarantees to multimedia connections in satellite networks. By implementing an adaptive transmission mechanism, the inherent challenge of mitigating the effect of rain attenuation on transmitted signals can be addressed. It has been shown that resource allocation and utilization can be made more efficient through prediction of the state of the channel at regular intervals within the duration of the lifetime of an accepted connection. In fact, we have shown that the frequent prediction of the attenuation experienced on the link improves the overall network's performance. Although increasing the frequency of the prediction of channel conditions improves the efficiency of transmission and utilization of resources, latency may be introduced to the link as the number of prediction periods increases. Thus, an optimal number of intervals for which the channel states should be predicted must be determined in order to minimize this latency. Based on simulation results, a four-period prediction is determined to be optimal. In our future work, we shall consider the determination of the optimal number of prediction periods analytically.

\section{Data Availability}

The results presented in the research were obtained from computer simulations. 


\section{Conflicts of Interest}

The authors declare that they have no conflicts of interest.

\section{Acknowledgments}

The authors like to thank the Center for Radio Access and Rural Technologies (CRART) of the University of KwaZuluNatal, Durban, 4041, South Africa, for supporting this work.

\section{References}

[1] G. Giambene, Resource Management in Satellite Networks: Optimization and Cross-Layer Design, Springer Science \& Business Media, Berlin, Germany, 2007.

[2] L. De Montera, L. Barthès, P. Golé, T. Marsault, and C. Mallet, "Short-term prediction of atmospheric attenuation in $\mathrm{q}$ band from ka-band measurements on earth-to-satellite links," in Proceedings of the EuCAP 2009: 3rd European Conference on Antennas and Propagation, pp. 341-344, IEEE, Berlin, Germany, March 2009.

[3] J. X. Yeo, Y. H. Lee, and J. T. Ong, "Rain attenuation prediction model for satellite communications in tropical regions," IEEE Transactions on Antennas and Propagation, vol. 62, no. 11, pp. 5775-5781, 2014.

[4] A. Kelmendi, G. Kandus, A. Švigelj, A. Vilhar, A. Hrovat, and E. Leitgeb, "Evaluation of the Gaussian copula prediction method for joint rain attenuation statistics in earth-satellite site diversity systems," in Proceedings of the 2016 International Conference on Broadband Communications for Next Generation Networks and Multimedia Applications (CoBCom), pp. 1-6, IEEE, Graz, Austria, September 2016.

[5] D. Petraki, M. Anastasopoulos, and P. Cottis, "Call admission control in satellite networks under rain fading," IEEE Communications Letters, vol. 12, no. 5, pp. 377-379, 2008.

[6] D. K. Petraki, M. P. Anastasopoulos, A. V. Vasilakos, and P. G. Cottis, "A distributed call admission control scheme for satellite networks under rain fading," in Proceedings of the 2009 International Conference on Wireless Communications and Mobile Computing: Connecting the World Wirelessly, pp. 716-720, ACM, Leipzig, Germany, June 2009.

[7] M. Anastasopoulos, D. Petraki, A. Vasilakos, P. Cottis, and H.-H. Chen, "Call admission control scheme for multiclass services under rain fading for satellite networks," IEEE Transactions on Wireless Communications, vol. 8, no. 5, pp. 2473-2483, 2009.

[8] D. K. Petraki, M. P. Anastasopoulos, H.-H. Chen, and P. G. Cottis, "Distributed resource allocation for delay-sensitive services in satellite networks using game theory," IEEE Transactions on Computational Intelligence and AI in Games, vol. 1, no. 2, pp. 134-144, 2009.

[9] B. Grémont and A. Tawfik, "Markov modelling of rain attenuation for satellite and terrestrial communications," in Proceedings of the Twelfth International Conference on Antennas and Propagation (ICAP 2003), Exeter, UK, March 2003.

[10] L. Castanet, T. Deloues, and J. Lemorton, "Channel modelling based on n-state markov chains for satcom systems simulation," in Proceedings of the Twelfth International Conference on Antennas and Propagation, 2003 (ICAP 2003), Exeter, UK, March 2003.

[11] L. Csurgai-Horváth, "Markovian properties of the q-band satellite channel with rain attenuation," in Proceedings of the
2016 10th European Conference onAntennas and Propagation (EuCAP), pp. 1-5, IEEE, Davos, Switzerland, April 2016.

[12] F. Guidolin, M. Nekovee, L. Badia, and M. Zorzi, "A cooperative scheduling algorithm for the coexistence of fixed satellite services and 5G cellular network," in Proceedings of the 2015 IEEE International Conference on Communications (ICC), pp. 1322-1327, IEEE, London, UK, June 2015.

[13] F. Guidolin and M. Nekovee, "Investigating spectrum sharing between 5G millimeter wave networks and fixed satellite systems," in Proceedings of the 2015 IEEE Globecom Workshops (GC Wkshps), pp. 1-7, IEEE, San Diego, CA, USA, December 2015.

[14] J. Zhang, B. Evans, M. A. Imran, X. Zhang, and W. Wang, "Performance analysis of $\mathrm{C} / \mathrm{U}$ split hybrid satellite terrestrial network for 5G systems," in Proceedings of the 2015 IEEE 20th International Workshop on Computer Aided Modelling and Design of Communication Links and Networks (CAMAD), pp. 97-102, IEEE, Guildford, UK, September 2015.

[15] M. Imran, J. Zhang, B. Evans, X. Zhang, and W. Wang, "Green hybrid satellite terrestrial networks: fundamental trade-off analysis," in Proceedings of the 2016 IEEE 83rd Vehicular Technology Conference, Nanjing, China, May 2016.

[16] Y. Vasavada, R. Gopal, C. Ravishankar, G. Zakaria, and N. BenAmmar, "Architectures for next generation high throughput satellite systems," International Journal of Satellite Communications and Networking, vol. 34, no. 4, pp. 523-546, 2016.

[17] P. T. Mathiopoulos, E. A. Candreva, A. B. Awoseyila et al., "Performance improvement techniques for the DVB-RCS2 return link air interface," International Journal of Satellite Communications and Networking, vol. 33, no. 5, pp. 371-390, 2015.

[18] G. Araniti, M. Condoluci, A. Orsino, A. Iera, and A. Molinaro, "Effective resource allocation in 5G-satellite networks," in Proceedings of the 2015 IEEE International Conference on Communications (ICC), pp. 844-849, IEEE, London, UK, June 2015.

[19] G. Araniti, A. Orsino, J. Cosmas, A. Molinaro, and A. Iera, “A low computational-cost subgrouping multicast scheme for emerging 5G-satellite networks," in Proceedings of the 2016 IEEE International Symposium on Broadband Multimedia Systems and Broadcasting (BMSB), pp. 1-6, IEEE.

[20] P. Pace, G. Aloi, and S. Marano, "Efficient real-time multimedia connections handling over DVB-RCS satellite system," in Proceedings of the IEEE Global Telecommunications Conference, 2004. GLOBECOM'04, vol. 5, pp. 2722-2727, IEEE, Dallas, TX, USA, December 2004.

[21] F. De Rango, M. Tropea, P. Fazio, and S. Marano, "Call admission control with statistical multiplexing for aggregate MPEG traffic in a DVB-RCS satellite network," in Proceedings of the GLOBECOM'05. IEEE Global Telecommunications Conference, 2005, vol. 6, p. 6, December 2005.

[22] P. Pace and G. Aloi, "Effective admission policy for multimedia traffic connections over satellite DVB-RCS network," ETRI Journal, vol. 28, no. 5, pp. 593-606, 2006.

[23] O. E. Imole and T. Walingo, "Call admission control for weather-impacted multimedia satellite networks," in Proceedings of the 2017 IEEE 85th Vehicular Technology Conference (VTC Spring), pp. 1-5, IEEE, Sydney, NSW, Australia, June 2017.

[24] ETSI, "Digital Video Broadcasting (DVB); second generation framing structure, channel coding and modulation systems for broadcasting, interactive services, news gathering and other broadband satellite applications, technical report, 
technical report," Technical Reports, ETSI, Sophia Antipolis, France, 2009.

[25] ETSI EN 301, Second Generation DVB, Interactive Satellite System (Dvb-Rcs2); Part 2: Lower Layers for Satellite Standard, Digital Video Broadcasting (DVB) Std.ETSI, Sophia Antipolis, France, 2012.

[26] T. J. O. Afullo, "Raindrop size distribution modeling for radio link design along the eastern coast of South Africa," Progress In Electromagnetics Research B, vol. 34, pp. 345-366, 2011.

[27] A. A. Alonge and T. J. Afullo, "Regime analysis of rainfall drop-size distribution models for microwave terrestrial networks," IET Microwaves, Antennas \& Propagation, vol. 6, no. 4, pp. 393-403, 2012.

[28] A. A. Alonge, Queueing theory approach to rain fade analysis at microwave and millimeter bands in tropical africa, Ph.D. thesis, University of KwaZulu-Natal, Durban, South Africa, 2014.

[29] M. N. Ahuna, T. J. O. Afullo, and A. Alonge, "Outage prediction during intense rainstorm events using queuing theory and Markov chains over radio links," Progress In Electromagnetics Research, vol. 73, pp. 183-196, 2018.

[30] B. Maruddani, A. Kurniawan, S. Sugihartono, and A. Munir, "Rain fade modelling using hidden markov model for tropical area," in Proceedings of the Progress in electromagnetics Research Symposium (PIERS), pp. 96-100, Cambridge, MA, USA, July 2010.

[31] F. D. Diba, T. J. Afullo, and A. A. Alonge, “Time series rainfall spike modelling from Markov chains and queueing theory approach for rainfall attenuation over terrestrial and earthspace radio wave propagation in jimma, Ethiopia," in Proceedings of the 2016 Progress In Electromagnetic Research Symposium (PIERS), pp. 4991-4995, IEEE, Shanghai, China, August 2016.

[32] A. E. Drougas, A. D. Panagopoulos, and P. G. Cottis, "Stochastic verification of the first-order markovian assumption of rain attenuation for satellite channel dynamic modeling," IEEE Communications Letters, vol. 12, no. 9, pp. 663-665, 2008.

[33] L. Chen, B. Wang, X. Chen, X. Zhang, and D. Yang, "Utilitybased resource allocation for mixed traffic in wireless networks," in Proceedings of the 2011 IEEE Conference on Computer Communications Workshops (INFOCOM WKSHPS), pp. 91-96, IEEE, Shanghai, China, April 2011. 


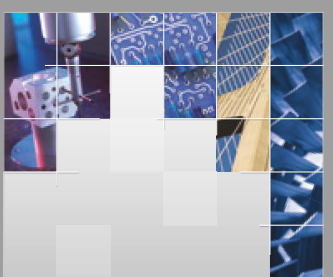

\section{Enfincering}
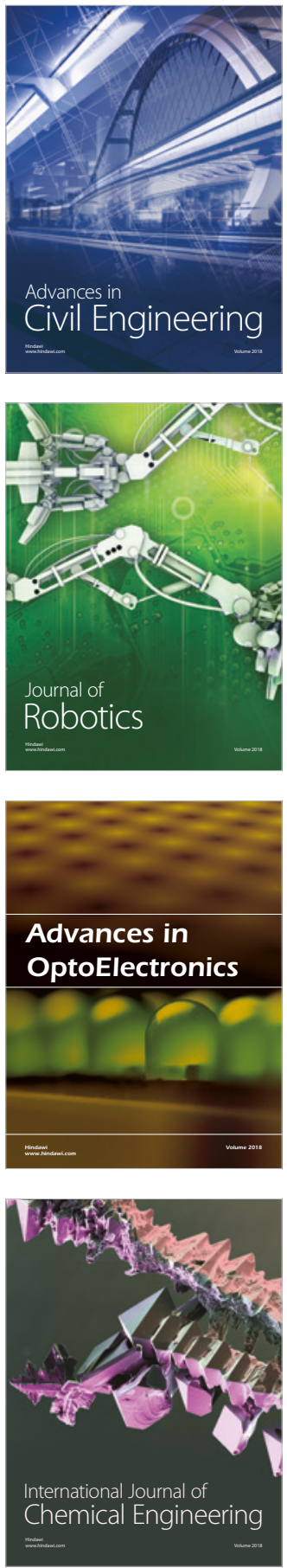



\section{Rotating \\ Machinery}

The Scientific World Journal

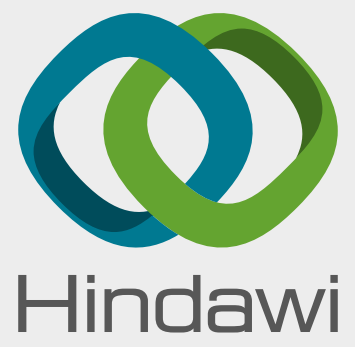

Submit your manuscripts at

www.hindawi.com



\section{Advances \\ Multimedia}
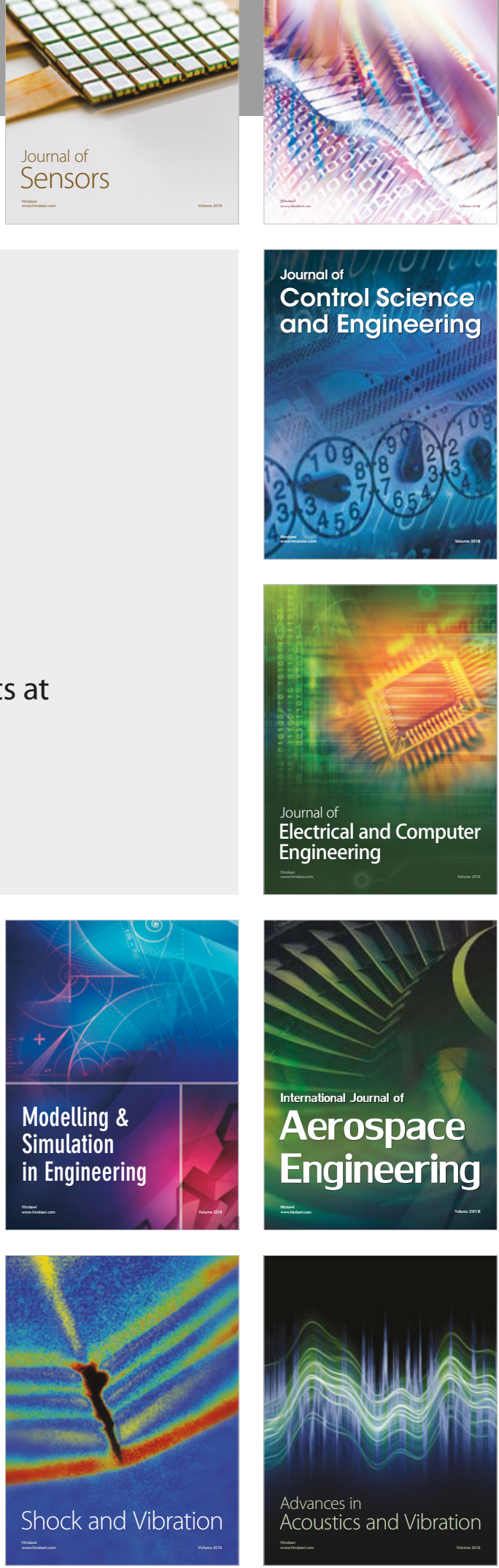